\title{
Corrole-based Nanostructures for Sensing Applications
}

\author{
Roberto Paolesse ${ }^{1}$, Luca Tortora ${ }^{1}$, Giuseppe Pomarico ${ }^{1}$, Sara Nardis ${ }^{1}$, Alexandro Catini ${ }^{2}$, Arnaldo \\ D'Amico' ${ }^{2}$ Corrado Di Natale ${ }^{2}$ \\ ${ }^{1}$ Dipartimento di Scienze e Tecnologie Chimiche, Università di Roma Tor Vergata, via della Ricerca \\ Scientifica 1, 00133 Rome, Italy \\ roberto.paolesse@uniroma2.it \\ ${ }^{2}$ Dipartimento di Ingegneria Elettronica, Università di Roma Tor Vergata, via del Politecnico, 00133
}

Rome, Italy

\begin{abstract}
:
Porphyrin and related macrocycles have been widely exploited as sensing materials in chemical sensors. The performances of such materials are generally depicted in terms of the binding properties of the individual molecule, but we have recently showed that the supramolecular aggregation of the macrocycles in the solid films could offer additional binding mechanisms, enhancing the sensing properties of the material. Among the different porphyrin analogs, corrole has attracted an increasing interest, due to its peculiar properties, demonstrated as sensing material in the case of carbon monoxide detection. We report here the sensing performances of corrole aggregates deposited to functionalize quartz crystal microbalances. The porous nanostructures of the organic framework offer additional binding mechanisms not available to the individual receptor, so allowing enhanced performances as sensing material.
\end{abstract}

Key words:porphyrin, corrole, quartz microbalances, organic framework, supramolecular aggregates.

\section{Introduction}

The controlled organization of functional heterocyclic chromophores, such as porphyrinoids and alike, into highly ordered selfassembled arrays is an appealing area of research with great potential in sensor applications. We have recently demonstrated that the sensing properties are not limited to the single porphyrin molecule [1]; in chemical sensors the sensing element is usually deposited as thin layer and in this case the supramolecular aggregation modes could further enrich the sensing mechanism of porphyrin derivatives, offering binding interaction not possible to the single porphyrin unit [2]. In particular we observed unexpected high sensitivities of porous dihydroxyphenylporphyrin layers towards $\mathrm{CO}$ detection, even in the absence of coordination to the individual porphyrin unit [1].

This approach could be particularly promising for sensing applications and we have been interested in the scope of such methodology for other pyrrolic macrocycles. Among the wide family of porphyrinoids, corrole (figure 1) has recently assumed a protagonist role, due to the peculiar properties showed by this macrocycle [3].

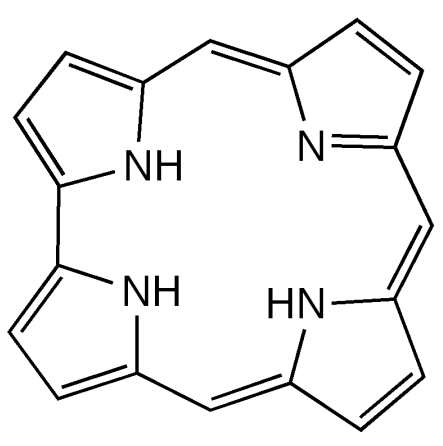

Fig. 1. Molecular structure of corrole.

We have prepared different metal complexes of dihydroxyphenyl substituted corroles and we have deposited thin film of such complexes onto quartz crystal microbalances (QMB) by spray coating technique. In the array we have also used a not functionalized corrole complex and the corresponding dihydroxyphenylporphyrins in order to appreciate the influence of the supramolecular motif in the sensing mechanism of the corrole layers. In figure 2 the structure of these compounds have been reported. 


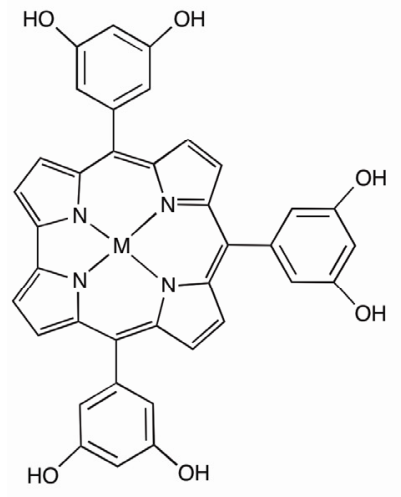

$\mathrm{M}=\mathrm{H}_{3}, \mathrm{FeCl}, \mathrm{Cu}$
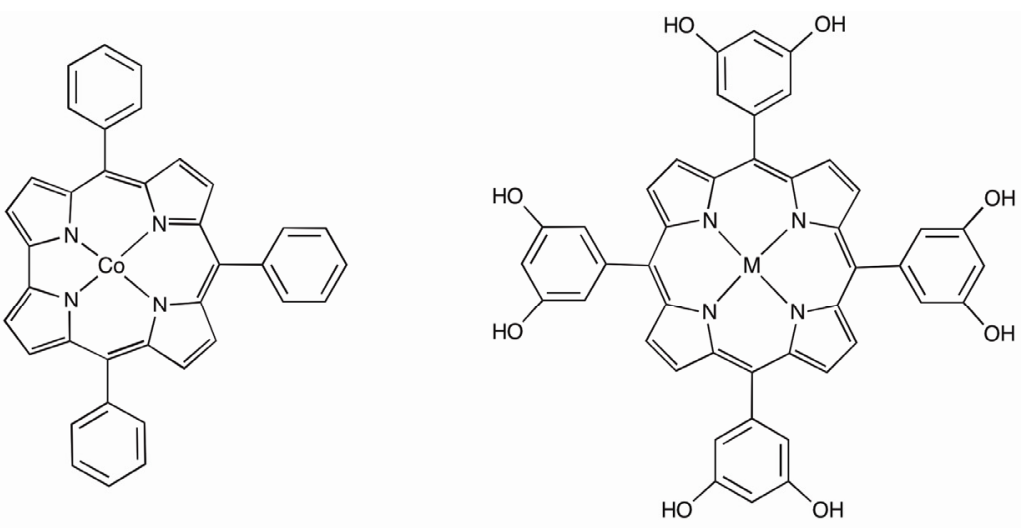

$\mathrm{M}=\mathrm{H}_{2}, \mathrm{FeCl}, \mathrm{Cu}$

Fig. 2. Molecular structures of the porphyrinoids used as sensing materials.

\section{Experimental}

QMB were AT-cut quartzes (Electroquartz) oscillating in the thickness shear mode (TSMR), with a fundamental frequency of $20 \mathrm{MHz}$; the quartz crystal diameter is $7.0 \mathrm{~mm}$, the gold electrodes diameter is $5.0 \mathrm{~mm}$. Thin films of sensing materials were deposited by spraycoating technique on both sides of TSMR quartz disks from $10^{-3} \mathrm{~mol} / \mathrm{L} \mathrm{CHCl}_{3}$ or $\mathrm{CHCl}_{3} / \mathrm{CH}_{3} \mathrm{OH}$ (1:1)solutions.

Vapors of the different analytes were generated by bubbling a $\mathrm{N}_{2}$ stream into a liquid sample of the compounds. The concentration of the volatile organic compound (VOC) in these saturated vapours was calculated by the Antoine's law [4]. These saturated vapors were diluted with nitrogen and fluxed into the sensor chamber by a computer-driven 4 channel massflow controller (MKS). The flow rate was kept at the constant value of $200 \mathrm{~cm}^{3} / \mathrm{min}$. Nitrogen oxide and carbon monoxide diluted in nitrogen certified tank (500 ppm, Rivoira) was used as $\mathrm{NO}$ and $\mathrm{CO}$ sources; the different gas concentrations were obtained by dilution of these gas mixtures with nitrogen and then fluxed by the same mass-flow controller into the measurement chamber. Carbon dioxide tank was used and diluted with nitrogen as in the case of $\mathrm{CO}$.

The developed sensors have been housed into the measurement chamber of the Tor Vergata electronic nose and they were exposed to different concentrations of both gases and volatile organic compounds (VOC) vapors. These gases and VOCs were chosen as model analytes to test the selectivity properties of the developed sensor array.

\section{Results and Discussion}

The QMB functionalized with porphyrin and corrole derivatives showed very fast responses upon exposure to both VOC and gases, indicating good diffusion rates of the analytes inside the thin films, as expected for porous layers, and confirming the efficient action of the peripheral hydroxy groups in the construction of the porous film, also in the case of corrole derivatives.

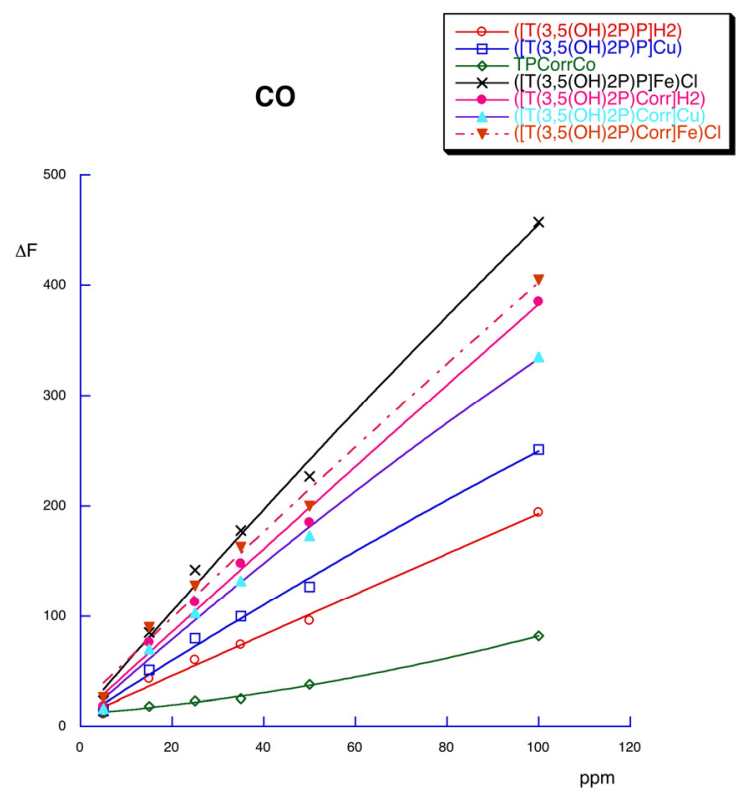

Fig. 3. Adsorption isotherms of $Q M B$ sensors toward $\mathrm{CO}$.

The QMBs functionalized with hydroxysubstituted corroles showed a sensitivity to CO similar to that already observed for similar porphyrin derivatives. The $\mathrm{CO}$ binding isotherm is reported in figure 3; the high sensitivity observed for the detection of carbon monoxide, confirm that the organic framework formed by 
the corrole rings can efficiently entrap $\mathrm{CO}$. This behavior is also confirmed with NO, which is a gas having molecular dimensions similar to that of $\mathrm{CO}$.

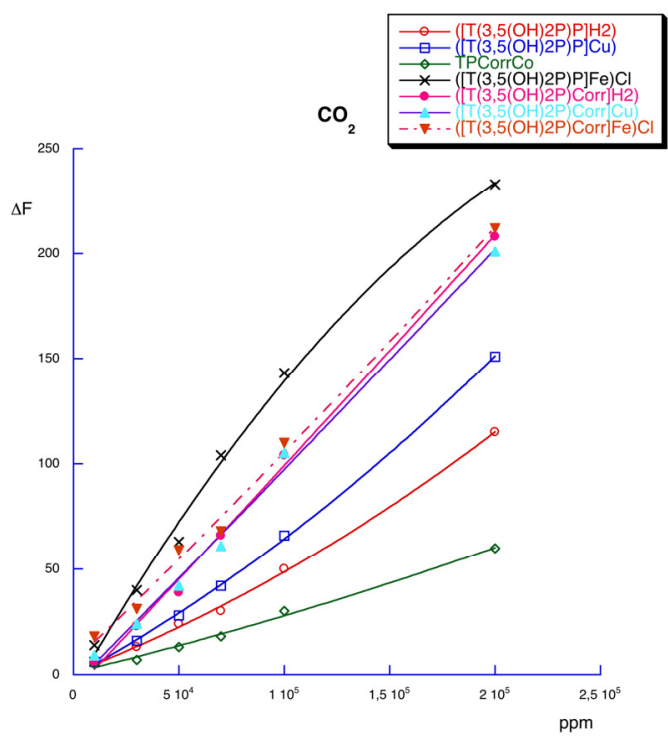

Fig. 4. Adsorption isotherms of $Q M B$ sensors toward $\mathrm{CO}_{2}$.

It is interesting to observe the low response observed in the case of the non-functionalized corrole, where the coordination to the metal center is the corresponding interaction mechanism [5]. From the comparison of the different responses it is possible to appreciate the influence of the different sensing mechanism on the overall sensor response.

In the case of $\mathrm{CO}_{2}$ (figure 4), on the other hand, we observed a significantly lower sensitivity than that observed for $\mathrm{CO}$ and $\mathrm{NO}$, confirming that the molecular dimension is the critical parameters for the sensing performances. However, also in this case the nonfunctionalized corrole showed the lowest response, probably due to the more compact framework that this compound forms in the solid state.

In the case of VOC, a completely different behavior was observed. The sensitivity to non polar VOCs, such as for example hexane, was greatly depressed to respect the non functionalized corrole, which showed in this case the highest response (figure 5).

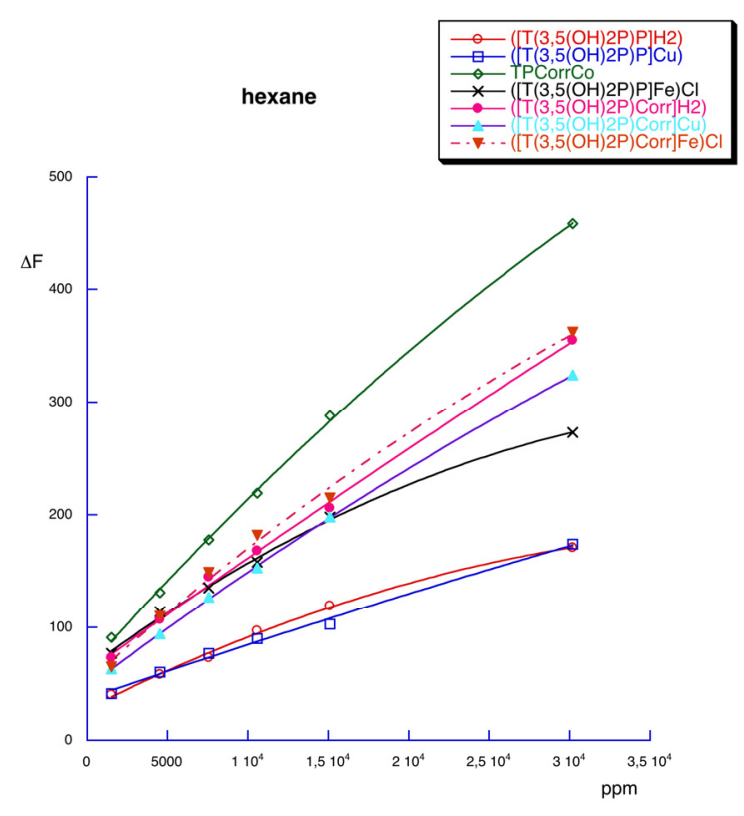

Fig. 5. Adsorption isotherms of $Q M B$ sensors toward hexane.

On the other hand, a completely different scenario was obtained with ethanol, where the hydroxy substituted corroles showed a high sensitivity toward ethanol detection, as expected, due to the possibility of hydrogen bond interactions with the peripheral $\mathrm{OH}$ groups present in the corrole ring (figure 6).

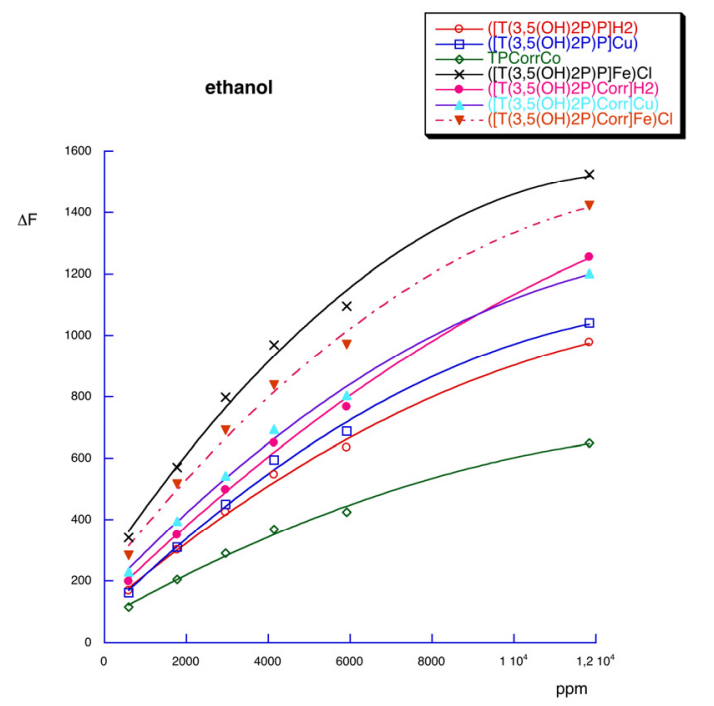

Fig. 6. Adsorption isotherms of $Q M B$ sensors toward ethanol.

This result is particularly interesting, because it can open a novel approach for the preparation of porphyrinoid layers having a completely different sensing behavior. In this case, the modification is operated on the peripheral substituents of the macrocycle, other than in the variation of the metal coordinated to the porphyrinoid ring. 


\section{Acknowledgements}

We gratefully acknowledge the support of MIUR, Italy (PRIN project 2009Z9ASCA).

\section{References}

[1] S. Nardis, G. Pomarico, L. Tortora, R. Capuano, A. D'Amico, C. Di Natale, R. Paolesse, J. Mater. Chem. 21, 18638-18644 (2011); doi: 10.1039/c1jm13623b

[2] C. Di Natale, D. Monti, R. Paolesse, Materials Today 13, 46-52 (2010); doi: 10.1016/S13697021(10)70127-9

[3] R. Paolesse, Synlett 15, 2215-2230 (2008); doi: 10.1055/s-2008-1078687.

[4] J. Riddick, A. Bunger, in Techniques of Chemistry, ed. A. Weissberger, Wiley Interscience, New York, 1970, Vol. II.

[5] J. M. Barbe, G. Canard, S. Brandès, R. Guilard, Chem. Eur. J. 13, 2118 (2007); doi: 10.1002/chem.200601143. 